\title{
Fine needle aspiration cytology is a valuable adjunct to axillary ultrasound in the preoperative staging of breast cancer
}

\author{
Morna MacNeill, ${ }^{1}$ Isobel Arnott, ${ }^{2}$ Jeremy Thomas ${ }^{1,3}$
}

'Department of Pathology, NHS Lothian, Western General Hospital, Edinburgh, UK ${ }^{2}$ Department of Radiology, NHS Lothian, Western General Hospital, Edinburgh, UK ${ }^{3}$ Breakthrough Breast Cancer Research Unit, Edinburgh, UK

\section{Correspondence to}

Dr Jeremy Thomas, Department of Pathology, Western General Hospital, Edinburgh EH4 2XU, UK; jeremy.thomas@luht.scot. nhs.uk

This paper was presented at the 1st British Breast Cancer Research Conference, Nottingham, UK, on 17 September 2010

Accepted 5 October 2010 Published Online First 19 November 2010

\section{ABSTRACT}

Aims To compare the predictive values of axillary ultrasound (US) combined with fine needle aspiration (FNA) cytology with tumour size (T stage) and grade in the preoperative staging of breast cancer. More precise definition of axillary FNA reporting nomenclature is also presented.

Patients and Methods 314 patients: 119 patients had suspicious US investigated by FNA, 195 patients had normal US not investigated further preoperatively. This study examined the node-positive and node-negative cases in these two groups, calculating predictive values for cytology, US, T stage and tumour grade, and tested comparisons for significance.

Results Axillary FNA has a positive predictive value of $84.8 \%$ compared with US (66.7\%). The difference is significant $(p=0.008)$. Negative US has a negative predictive value of $81.0 \%$ compared with a negative predictive value for cytology of $66.7 \%$, but the difference is not significant $(p=0.08)$. $43 \%$ of patients with unsatisfactory cytology were node positive. Of 195 patients with negative axillary US, 37 (19\%) had metastatic nodal disease. Fewer than $20 \%$ of these patients had micrometastases alone. Tumour size and grade influenced node status in US-suspicious cases only.

Conclusion Axillary FNA adds significantly to the positive predictive value provided by US. US gives falsenegative results in $19 \%$ of cases and only a small proportion of these can be explained by micrometastases. Unsatisfactory cytology needs to be repeated because of a high rate of positive nodes in this group.

\section{INTRODUCTION}

Management of the axilla is a critical part of effective management of patients with breast cancer. Previously, the decision to perform axillary node clearance (ANC) rather than sampling/ sentinel node biopsy was based largely on tumour size. Such selection methods resulted in a nodepositive rate of $65 \%$ for ANC patients and $35 \%$ for sample patients. ${ }^{1}$ Despite progress towards less invasive techniques, $5-10 \%$ of tumours $<0.5 \mathrm{~cm}$ in diameter and $10-20 \%$ of tumours $0.6-1 \mathrm{~cm}$ in diameter will be node positive, and $15-30 \%$ of clinically node-negative patients will develop axillary failure if the axilla is left untreated by either radiotherapy or dissection. ${ }^{2}$ There is a compelling clinical need to improve the accuracy of preoperative staging to ensure the most appropriate axillary surgery for the individual patient. The use of axillary ultrasound (US) coupled with targeted fine needle aspiration (FNA) cytology has increased in recent years in an attempt to satisfy this need. Since mid-2006 all patients with breast cancer treated at the Edinburgh Breast Unit have undergone axillary US as part of their preoperative management. The patients are from screening and symptomatic practices in the ratio of approximately 40:60.

This paper will: (1) review the efficacy of US in staging the axilla, and the contribution of axillary FNA to radiological staging data; (2) resolve ambiguities in trying to apply current breast FNA reporting nomenclature to axillary cytology; and (3) by reviewing a large number of US-negative cases, place the contribution of axillary US and FNA in context with the predictive value of tumour size and grade and node status.

\section{PATIENTS AND METHODS}

We reviewed the Western General Hospital Mammography Department records for the years 2006-7 to identify patients with breast cancer who had undergone axillary staging US. Those patients with suspicious US were examined further by FNA cytology with samples taken by the radiologist under US guidance. There was no immediate review of specimens for sample adequacy. All positive cytology samples were double-read, as has been local policy for many years. One hundred and nineteen patients with suspicious US and 195 patients with negative US were available for pathological review. During the period of study, sentinel node biopsy was carried out routinely to stage the axilla in the absence of preoperatively diagnosed nodal metastatic disease. Enhanced detection methods were not used and nodes were subjected to routine sectioning and $H \& E$ staining.

Pathological review included the recording of tumour type, pathological size and grade, axillary procedure and node status, and where nodes were positive the number of involved nodes and the size of metastases. The distribution of tumour type in the two groups of patients is summarised in table 1 . Where there was a mismatch between the FNA and final histological diagnosis, the FNA and lymph node histology were reviewed if required. Tumour grade was determined using Elston and Ellis's modification of Bloom and Richardson's method. ${ }^{3}$ Statistical significance was assessed with the $\chi^{2}$ test and logistic regression analysis was carried out using Analyse It v 2.01 for Microsoft Excel 2003. We accepted a value of $p=<0.05$ as significant. 
Table 1 Breakdown of US-negative and US-suspicious cases by tumour type

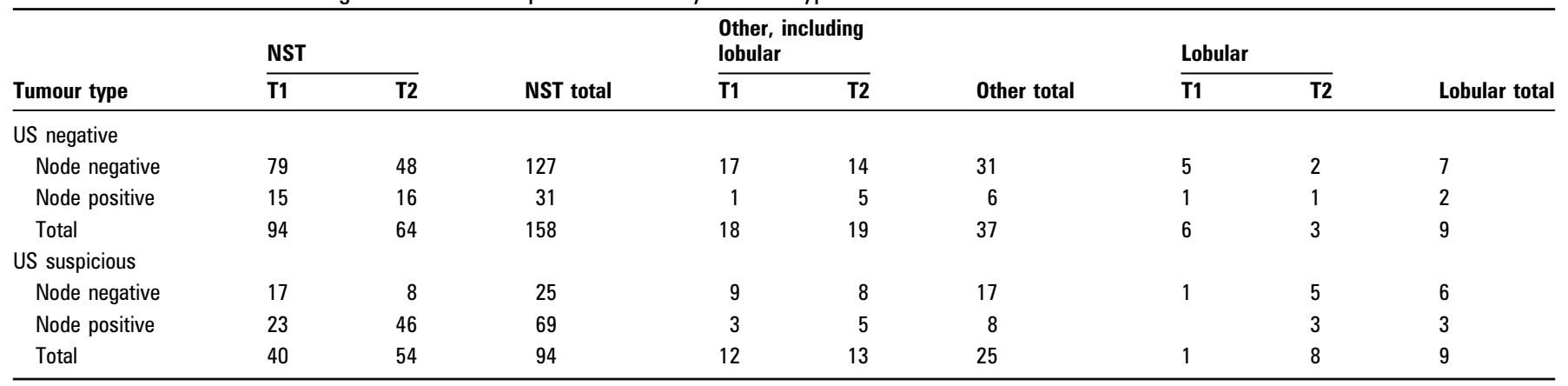

NST, no special type; US, ultrasound.

Criteria used to define suspicious features on ultrasound were as follows: (1) nodal cortical thickening $>2 \mathrm{~mm}$; (2) eccentric cortical thickening giving the appearance of a bulge; (3) anechoic areas in a node indicating possible replacement type metastases; (4) destruction of the outer capsule, the node no longer having a smooth outline.

We adapted current breast cytology reporting nomenclature ${ }^{4}$ for reporting axillary nodes: C1n, unsatisfactory, no malignant cells seen, no lymphoid cells seen; C2n, no malignant cells seen, lymphocytes present in moderate numbers; C3n, suspicious cells seen; C4n, suspicious cells seen, probably malignant; C5n, malignant cells seen.

We have used the following nomenclature for describing nodal metastases: macrometastasis is a nodal metastasis $>2 \mathrm{~mm}$, and micrometastasis is a nodal metastasis $>0.2 \mathrm{~mm}$ and $\leq 2 \mathrm{~mm}$.

\section{RESULTS}

\section{Patients with suspicious axillary US}

Positive FNA cytology (C5n)

The correlation between axillary FNA cytology and final lymph node status is summarised in table 2 . Axillary FNA with suspicious US showed a positive predictive value (PPV) of $84.8 \%$ and a negative predictive value (NPV) of $66.7 \%$. Of 77 node-positive cases where cytology had been performed, 66 underwent axillary clearance while the remaining 11 underwent node sampling and/ or sentinel node biopsy. Ten cases had positive cytology that was unconfirmed histologically (nine clearances and one sample/ sentinel node) but six of these had received neoadjuvant chemotherapy and showed node scarring indicative of a tumour response. A review of the cytology of the remaining four cases (JT) confirmed the presence of malignant cells, indicating that either the positive node had not been retrieved or the deposit had not been identified in the sections examined. One of these four remaining unconfirmed cases was treated by node sampling/sentinel node biopsy.

Table 2 Suspicious axillary ultrasound: correlation between axillary FNA cytology and final lymph node status

\begin{tabular}{lll}
\hline & Node status & \\
\cline { 2 - 3 } FNA result & Positive & Negative \\
\hline C1n & 10 & 13 \\
C2n & 9 & 18 \\
C3n & 1 & 1 \\
C4n & 1 & 0 \\
C5n & 56 & $10^{*}$ \\
Total & 77 & 42 \\
\hline *Includes six post-chemotherapy cases. \\
FNA, fine needle aspiration.
\end{tabular}

Unsatisfactory, negative and suspicious FNA cytology with positive histology (C1n, C2n, C3n and C4n)

Ten patients received C1n FNA diagnoses: six were repeated or had axillary core biopsy and returned suspicious results, four were not repeated. Nine patients received false-negative benign cytology diagnoses (C2n) with positive axillary histology. Three patients received suspicious (C3n or C4n) FNA diagnoses, two of whom had node metastases on histology.

Unsatisfactory or negative cytology with negative histology:

Thirteen patients received C1n FNA diagnoses with subsequent negative histology: 10 were treated by sampling/sentinel node biopsy, and 3 were treated by by node clearance. Eighteen patients had C2n FNA diagnoses with negative histology: 17 treated by node sampling/sentinel node biopsy and 1 by node clearance

The PPVs and NPVs referred to are all calculated from the original cytology (C5n and C2n values) as shown in table 2.

\section{Patients with negative axillary US}

Of the 195 patients with negative axillary US, 37 (19\%) had metastatic nodal disease on histology: 6 of these patients $(16 \%)$ had micrometastases only, 7 patients (19\%) had solitary replacement metastases, 12 patients (32\%) had a mixture of replacement and micrometastases, and 12 patients (32\%) had multiple replacement metastases. Of the 37 patients with metastatic disease, 21 (57\%) had T2/T3 tumours, range 21-75 mm, median $27 \mathrm{~mm}$.

\section{Tumour size, grade and US and relation to node status}

In a logistic regression analysis of $\mathrm{T}$ stage ( $\mathrm{T} 2 / 3$ versus $\mathrm{T} 1$ ), tumour grade and US and their relation to node status, all three variables were significantly independently predictive of node status ( $p=0.0002,0.0042$ and $<0.0001$ respectively). FNA cytology was also considered in the US-suspicious group and was the only significant factor in this group $(p=<0.0001)$.

We compared the node-positive/node-negative values for $\mathrm{T} 2 / 3$ tumours with T1 tumours in the US-negative and US-suspicious groups respectively using the $\chi^{2}$ test. In the US-negative group the difference was not significant $(p=0.052)$ whereas in the USpositive group it was significant $(\mathrm{p}=0.006)$.

We compared the node-positive/node-negative values for grade $2 / 3$ tumours with grade 1 tumours in the US-negative and USsuspicious groups respectively using the $\chi^{2}$ squared test. In the US-negative group the difference was not significant $(p=0.2)$ whereas in the US-positive group it was significant $(p=0.009)$.

The data for tumour size as expressed by tumour, node, metastases (TNM) T stage, grade and US evaluation and node status are summarised in tables 3-5. 
Table 3 Normal axillary ultrasound: relationship of tumour size (T stage) and grade to node status

\begin{tabular}{|c|c|c|c|c|c|c|c|c|c|}
\hline \multirow[b]{3}{*}{ Node status } & \multicolumn{9}{|c|}{ Ultrasound negative } \\
\hline & \multicolumn{4}{|l|}{ T1 } & \multicolumn{4}{|c|}{$\mathrm{T} 2$ and $\mathrm{T} 3$} & \multirow[b]{2}{*}{$\mathrm{T} 1+\mathrm{T} 2$ total } \\
\hline & Grade 1 & Grade 2 & Grade 3 & Total & Grade 1 & Grade 2 & Grade 3 & Total & \\
\hline Node positive, n (\%) & $1(4)$ & $9(17)$ & $6(19)$ & 16 & $3(43)$ & $13(28)$ & $5(17)$ & 21 & 37 \\
\hline Total, n (\%) & $28(25)$ & 53 (47) & $31(28)$ & 112 & 7 (8) & 47 (57) & $29(35)$ & 83 & 195 \\
\hline
\end{tabular}

Table 4 Suspicious axillary ultrasound: relationship of tumour size (T stage) and grade to node status

\begin{tabular}{|c|c|c|c|c|c|c|c|c|c|}
\hline \multirow[b]{3}{*}{ Node status } & \multicolumn{9}{|c|}{ Ultrasound suspicious } \\
\hline & \multicolumn{4}{|l|}{ T1 } & \multicolumn{4}{|c|}{$\mathrm{T} 2$ and $\mathrm{T} 3$} & \multirow[b]{2}{*}{$\mathrm{T} 1+\mathrm{T} 2$ tota } \\
\hline & Grade 1 & Grade 2 & Grade 3 & Total & Grade 1 & Grade 2 & Grade 3 & Total & \\
\hline Node positive, n (\%) & 0 & $15(60)$ & $11(61)$ & 26 & $2(100)$ & $22(67)$ & $27(87)$ & 51 & 77 \\
\hline Total, n (\%) & $6(12)$ & $25(51)$ & $18(37)$ & 49 & $2(3)$ & $33(50)$ & $31(47)$ & 66 & 115 \\
\hline
\end{tabular}

Four cases had no grade or size because of no residual disease post chemotherapy.

\section{Comparison of FNA cytology and US alone as predictors of node status}

The PPVs and NPVs for C5n versus C2n FNA and US-suspicious versus US-negative were compared by $\chi^{2}$ testing. The PPV for FNA was significantly higher than for US $(p=0.008)$ but the NPV for US was not significantly higher than that for FNA $(\mathrm{p}=0.08)$

The influence of the different variables studied above on PPVs and NPVs is summarised in table 6.

\section{DISCUSSION}

Although our data are in agreement with those previously reported we are not aware of such data being compared with the outcome of patients with normal axillary US. Our results are comparable with those reported by other groups in terms of sensitivity, specificity and predictive values. ${ }^{5}{ }^{6}$ The results will inevitably be influenced by the threshold of suspicion relating to the US technique. We are also aware that we studied this group of patients during an early part of our experience with this technique and that our performance, both radiological and cytological, is likely to have improved since.

A negative axillary US was followed by positive nodal histology in 37 cases; 18 of these cases had micrometastases $(\leq 2 \mathrm{~mm})$ either alone or in combination, while the remainder had metastases all of which were $>2 \mathrm{~mm}$. It is apparent that

Table 5 Relationship between tumour size (T stage) and grade on node status: all cases

\begin{tabular}{lllll}
\hline \multirow{2}{*}{ Node status } & \multicolumn{4}{l}{ Tumour size (T stage): T1 } \\
\cline { 2 - 5 } Grade 1 & Grade 2 & Grade 3 & Total \\
\hline Node negative, n (\%) & $33(97)$ & $54(68)$ & $32(67)$ & $119(74)$ \\
Node positive, n (\%) & $1(3)$ & $24(32)$ & $17(33)$ & $42(26)$ \\
Total & $34(21)$ & $78(48)$ & $49(31)$ & 161 \\
& Tumour size & $(\mathrm{T}$ stage): T2 and T3 & \\
Node negative, n (\%) & $4(44)$ & $45(56)$ & $28(46)$ & $77(52)$ \\
Node positive, n (\%) & $5(56)$ & $35(44)$ & $32(54)$ & $72(48)$ \\
Total & $9(6)$ & $81(54)$ & $61(41)$ & 149 \\
& All cases & & & \\
Node negative, n (\%) & $37(86)$ & $99(62)$ & $60(54)$ & $196(63)$ \\
Node positive, n (\%) & $6(14)$ & $59(38)$ & $49(46)$ & $114(37)$ \\
Total & $43(14)$ & $160(52)$ & $111(36)$ & 310 \\
\hline
\end{tabular}

Three T1 cases and one T2/3 (post-chemotherapy) case were ungraded. only a small proportion (16\%) of our false-negative US cases could be explained by micrometastatic disease alone. It is also important to appreciate the size of primary tumour associated with negative axillary US but positive nodes. T2 and T3 tumours were present in $57 \%$ of these cases. It raises the serious point that $\mathrm{T}$ stage information should be factored into the interpretation of axillary US findings and the management of the axilla based on them, particularly in US-suspicious cases.

Suspicious axillary US has a PPV of $67.0 \%$. In the presence of negative or unsatisfactory cytology, up to one-third of patients can be expected to have nodal disease. A negative FNA (C2n) is followed by positive histology in 33\% of cases (9/27), while unsatisfactory cytology $(\mathrm{C} 1 \mathrm{n})$ is followed by positive histology in $43 \%$ of cases $(10 / 23)$. We are aware of a relatively high apparently false-positive rate in our series (10 out of 66 cases). We reviewed the six chemotherapy cases and identified node scarring in this group indicating a tumour response in lymph nodes. Four cases remain unexplained due to either a failure to retrieve the node by the surgeon or to detect the metastasis by the pathologist. Review of the individual FNAs confirmed the presence of malignant cells in all cases.

In the presence of suspicious axillary US in our breast unit, a decision to carry out axillary clearance rather than a more limited sampling/sentinel node procedure is largely based on the results of cytology. Is this justified? Positive cytology has a high PPV (84.8\%). Negative cytology has a NPV of $66.7 \%$. From our data, adding cytology to axillary US does improve the accuracy of patient selection for axillary node clearance by increasing the PPV from $67 \%$ to $84.8 \%$, which is a significant difference $(p=0.008)$.

Hinson and colleagues have further refined this approach to patient selection for ANC by stratifying patients according to

Table 6 Influence of cytology, T stage and US status on PPV and NPV

\begin{tabular}{lll}
\hline Variable & PPV, \% (95\% CI) & NPV, \% (95\% Cl) \\
\hline C5n versus C2n & $84.8(76.2$ to 93.5$)$ & $66.7(55.3$ to 78.0$)$ \\
T2/3 cases: US-suspicious versus & $77.3(67.2$ to 87.4$)$ & $74.7(64.2$ to 85.2$)$
\end{tabular}

US-negative

US-suspicious T2/3 versus T1 $77.3(67.2$ to 87.4$) \quad 46.9$ (34.9 to 59.0$)$ US-suspicious versus US-negative: $\quad 67.0$ (58.4 to 75.6$) \quad 81.0$ (73.9 to 88.2 ) all cases

T2/3 versus T1: all cases $\quad 48.3(40.3$ to 56.3$) \quad 73.9(66.9$ to 81.0$)$ US-negative T2/3 versus T1 25.3 (15.9 to 34.7$) \quad 85.7$ (78.2 to 93.2) 
risk. ${ }^{7}$ They subdivided their patients into a low-risk group and a high-risk group on the basis of tumour grade, lymphovascular invasion and tumour size, and showed that $82 \%$ of low-risk patients were node negative as compared with $48 \%$ of high-risk patients

Koelliker and colleagues demonstrated a sensitivity of $71-75 \%$ and a specificity of $100 \%{ }^{8}$ They also showed increasing sensitivity with increasing tumour size. We used pathological tumour size and not the preoperative estimate of size (usually US). US data for tumour size were not available to us at the time of the analysis and there is a good evidence base that US and pathological tumour size measurements are in close agreement especially with T1 cancers. $^{9}$

Krishnamurthy showed a sensitivity of $84 \%$ and a specificity of $100 \%{ }^{10}$ All cases with three or more lymph nodes with metastatic disease, and $93 \%$ of those with a metastatic deposit measuring more than $0.5 \mathrm{~mm}$, were detected by US-guided FNA. The probability of detecting lymph nodes with smaller metastatic deposits measuring less than $0.5 \mathrm{~cm}$ was $44 \%$. The common causes of discrepancy between the initial and final axillary lymph node status include failure to visualise all lymph nodes during US examination, small-sized metastases, and preoperative neoadjuvant chemotherapy.

Jain found a sensitivity and specificity in line with our predictive values but also looked at the value of FNA of ultrasonically normal lymph nodes and found that the sensitivity dropped to $54 \%$ but the specificity remained very high at $100 \%$, and they suggested that US FNA of normal appearing nodes might be beneficial in cases where decisions regarding neoadjuvant chemotherapy would be affected by the results. None of the primary tumour features (histology, size, grade, vascular invasion, oestrogen/progesterone receptor status and Her-2-neu status) predicted concordance of US FNA and sentinel node biopsy/axillary clearance. ${ }^{6}$

Alkuwari and Auger noted that the sensitivity of FNA was higher in their axillary clearance group in comparison with their sentinel node biopsy group, and attributed this to the smaller size of metastatic deposits (median $0.25 \mathrm{~cm}$ ) in the latter group. ${ }^{11}$

Rao and colleagues have made a preliminary evaluation of the comparison between core needle biopsy and FNA in the investigation of the US-suspicious group and concluded that there were no data to support the use of core needle biopsy over FNA. However, their numbers were small. Sensitivities of the two techniques were comparable and FNA was considerably cheaper. ${ }^{12}$ The major saving generated by either technique however is related to one-step axillary surgery. ${ }^{5}$

Our study raises important questions about the nomenclature used in reporting axillary cytology: the most important area of potentially confused communication relates to the use and understanding of 'unsatisfactory' and 'negative' designations. The absence of lymphoid cells in the FNA signifies that the needle had not entered the lymph node and that the test could not be regarded as negative. It is notable that the small number of 'unsatisfactory' examinations that were followed by a repeat FNA or core biopsy resulted in suspicious or positive results from the repeated test. We have introduced the use of an 'n' suffix for all our axillary cytology reports to remove any ambiguity about the potentially different meaning in these reports in comparison to their breast counterparts. We are aware that the assessment of the lymphoid content in a nodal FNA can be formalised and may have the potential of standardising practice both within and between bresst units. ${ }^{13}$ We are reviewing our practice in the light of this.

\section{Take-home messages}

Adding cytology to axillary ultrasound (US) improves accuracy of selection for node clearance.

- T stage and higher histological grade is associated significantly with node status in US-suspicious cases only.

- A C1n fine needle aspiration needs to be repeated-it is not negative.

- Negative US is associated with positive nodal disease in $19 \%$ of cases-only a small proportion is explained by micrometastases.

\section{Interactive multiple choice questions}

- This JCP article has an accompanying set of multiple choice questions (MCOs). To access the questions, click on BMJ Learning: Take this module on BMJ Learning from the content box at the top right and bottom left of the online article. For more information please go to: http://jcp.bmj.com/education Please note: the MCOs are hosted on BMJ Learning - the best available learning website for medical professionals from the BMJ Group. If prompted, subscribers must sign into JCP with their journal's username and password. All users must also complete a one-time registration on BMJ Learning and subsequently log in (with a BMJ Learning username and password) on every visit.

\section{CONCLUSION}

Axillary US has a high PPV and NPV in staging the axilla and is improved significantly by adding information from FNA cytology. US gives false-negative results in 19\% of cases; only a small proportion of these $(16 \%)$ can be explained by micrometastatic disease. Further work is required to refine the accuracy of this valuable staging technique. We underline the need to take in to account tumour size when planning axillary surgery for breast cancer.

Acknowledgements The authors are grateful to Drs Jim Walsh and Melanie Smith who carried out many of the ultrasound examinations on patients in this study, and to Drs Joe Loane and Catriona Cowan who reported many of the FNAs. We are indebted to Dr Gill Kerr, statistician, NHS Lothian, for statistical advice.

\section{Competing interests None.}

Provenance and peer review Not commissioned; externally peer reviewed.

\section{REFERENCES}

1. Somner JEA, Dixon JMJ, Thomas JSJ. Node retrieval in axillary lymph node dissections: recommendations for minimum numbers to be confident about node negative status. J Clin Pathol 2004;57:845-8.

2. Singletary SE. Management of the axilla in early stage breast cancer. In: Perry MC, ed. American Society of clinical oncology educational book. Alexandria, VA: American Society of Clinical Oncology, 1998:132-41.

3. Elston CW, Ellis IO. Pathological prognostic factors in breast cancer (I): the value of histological grade in breast cancer: experience from a large study with long term follow up. Histopathology 1991;19:403-10.

4. Ellis I0, Humphreys S, Michell M, et al. Guidelines for non-operative diagnostic procedures and reporting in breast cancer screening: NHSBSP 50. Sheffield, UK: NHS Cancer Screening Programmes, 2001;50:20-2

5. Genta F, Zanon E, Camanni M. Cost/accuracy ratio analysis in breast cancer patients undergoing US-guided fine-needle aspiration cytology, sentinel node biopsy, and frozen section of node. World J Surg 2007;31:1153-4. 
6. Jain A, Haisfield -Wolfe ME, Lange J. The role of US-guided fine-needle aspiration of axillary nodes in the staging of breast cancer. Ann Surg Oncol 2008;15:462-71.

7. Hinson JL, McGrath P, Moore A, et al. The critical role of axillary us and aspiration biopsy in the management of breast cancer patients with clinically negative axilla. Ann Surg Oncol 2008;15:250-5.

8. Koelliker SL, Chung MA, Mainiero MB, et al. Axillary lymph nodes: US-guided fineneedle aspiration for initial staging of breast cancer-correlation with primary tumor size. Radiology 2008:246:81-9.

9. Tresserra F, Feu J, Grases PJ, et al. Assessment of breast cancer size: Sonographic and pathologic correlation. J Clin Ultrasound 1999;27:485-91.
10. Krishnamurthy S, Sneige N, Bedi DG, et al. Role of US-guided fine-needle aspiration of indeterminate and suspicious axillary lymph nodes in the initial staging of breast carcinoma. Cancer 2002;95:982-8.

11. Alkuwari $\mathbf{E}$, Auger M. Accuracy of fine-needle aspiration cytology of axillary lymph nodes in breast cancer patients: a study of 115 cases with cytologic-histologic correlation. Cancer 2008;114:89-93.

12. Rao R, Lilley L, Andrews V, et al. Axillary staging by percutaneous biopsy: sensitivity of fine-needle aspiration versus core needle biopsy. Ann Surg Oncol 2009;16:1170-5

13. Cameron SHE, Andrade SE, Pambuccian SE. Endobronchial ultrasound-guided transbronchial needle aspiration cytology: a state of the art review. Cytopathology 2010;21:6-26. 